\title{
Comparison of Endoloop and polymer locking clip in ligating appendiceal stump during laparoscopic appendectomy
}

\author{
Osman Şimşek, İsmail Ahmet Bilgin, Sezgin Uludağ, Fatih Dal, Mehmet Velidedeoğlu, \\ Kaya Sarıbeyoğlu, Salih Pekmezci \\ Emergency Surgery Unit, Department of General Surgery, Istanbul University Cerrahpasa Faculty of Medicine, Istanbul, Turkey
}

\begin{abstract}
Introduction: The aim of this study was to compare use of Endoloop (Ethicon, Inc., Somerville, NJ, USA) and Hem-o-lok (Teleflex, Inc., Wayne, PA, USA) polymer locking clip for closure of the appendix base during laparoscopic appendectomy.

Materials and Methods: In this prospective clinical study, 2 groups (Group 1: Endoloop; Group 2: Hem-o-lok) were formed and demographics, operating time, stump closure time, length of postoperative hospital stay, cost, and complications were compared.

Results: No perioperative or major postoperative complications were encountered. There was no mortality. Mean operating time was $50 \pm 10.6$ minutes for Group 1 and $40 \pm 12.6$ minutes for Group 2. Mean appendiceal base closure time was determined to be $187 \pm 102$ seconds and $69 \pm 30$ seconds in Group 1 and 2, respectively. Mean hospital stay was $53 \pm 33.4$ hours and $47.5 \pm 34$ hours for Group 1 and 2, respectively. Comparison of operating time revealed statistically significant difference $(t=3.22 ; p=0.002)$, and base closure time in Group 1 was significantly longer $(z=6.388 ; p<0.001)$. Remaining statistical comparisons did not reveal any statistically significant results.

Conclusion: Polymeric clip application of the appendiceal base had statistically significantly better results in terms of technical comfort and operating time in comparison with application of Endoloop. Further clinical studies are required to reach a final conclusion.
\end{abstract}

Keywords: Appendectomy; Endoloop; Hem-o-lok; laparoscopic appendectomy; polymeric clip.

\section{Introduction}

Appendectomy is the most effective treatment of acute appendicitis and mostly performed operation of emergency surgery worldwide. Since the introduction of laparoscopy, this minimally invasive technique was quickly adapted to appendectomy. Laparoscopic appendectomy (LA) is a safe and easy operation offering well-known advantages of laparoscopy, such as faster postoperative recovery, good cosmetics, less wound complications, less postoperative pain, and etc. ${ }^{[1,2]} \mathrm{A}$ recent meta-analysis comparing LA and open appendectomy (OA) has revealed that LA is 
associated with less postoperative pain, quicker postoperative rehabilitation, shorter hospital stay, and fewer postoperative complications than $\mathrm{OA} .{ }^{[3]}$ Moreover, LA is more advantageous in complicated appendicitis without any additional risk of intraabdominal abscess. This positive aspect has been shown by the systematic review of Markides G, et al. ${ }^{[4]}$

Another advantage of LA is the decrease of postoperative bowel obstruction rate, a benefit reported by Markar SR, et al. in their systematic review. ${ }^{[5]}$

LA technique has well-established steps and ligation of the base of the appendix is the critical maneuver since inappropriate closure may lead to severe postoperative surgical infections. Various techniques, including ligation by surgical knots, ENDOLOOP ${ }^{\circledR}$ Ligature (ETHICON; New Jersey, USA), surgical clips, endoscopic staplers, and etc. have been used so far in order to achieve appendix base closure. ${ }^{[6]}$

The results of our study, comparing ENDOLOOP ${ }^{\circledR}$ Ligature (ETHICON; New Jersey, USA) and polymer locking clip for the closure of the appendix base during LA, were reported in this study.

\section{Materials and Methods}

Patients who underwent laparoscopic appendectomy for acute appendicitis in Istanbul University, Cerrahpasa Medical Faculty, Emergency Unit between 01.01.2013 and 01.01.2014 were included into the study. Those who underwent appendectomy without a histopathological proof of acute appendicitis were excluded.

Eighty-seven patients with acute appendicitis underwent laparoscopic appendectomy. Appendix stumps were closed by ENDOLOOP ${ }^{\circledR}$ Ligature (ETHICON; New Jersey, USA) and Hem-o-lok ${ }^{\circledR}$ (Telefl ex Medical; New York City, USA) polymer locking clip in fifty-seven and thirty patients, respectively. In order to compare the groups equally, thirty patients were randomly selected in the "endo-loop group" with envelope manner. Two groups (Group 1: endo-loop and Group 2: locking clip) were formed and the results were compared regarding demographics, operating time, stump closure time, postoperative hospital stay, cost, and complications. The results were statistically evaluated by SPSS software and Pearson chi-square, Shapiro Wilk, Mann-Whitney U, and Aspin Welch tests were used.

\section{Surgical Technique}

All operations were performed under general anesthesia. The monitor was placed at patient's right and the surgical team, including the operating surgeon and camera assistant, stood at patient's left. A Foley catheter was placed in each patient and removed at the end of the operation. The operating time was started by the initial incision of the first port.

Laparoscopic appendectomy was performed through three ports. An infraumbilical $10 \mathrm{~mm}$ port was inserted by blind (Veress needle) in patients with no previous abdominal operations or by open (Hasson) technique in those with prior abdominal surgery. A 30 - laparoscope was used to explore the abdomen. The patients were put in slight Trendelenburg and left decubitus positions.

An additional second port of $10 \mathrm{~mm}$ was inserted under direct vision from left iliac fossa and the third port of 5 $\mathrm{mm}$ was placed above the pubis. The appendix was identified and freed from infl ammatory adhesions. The mesoappendix was divided by Ligasure (LigasureTM, Vessel Sealing System, Covidien, MA, USA).

Appendiceal base was ligated by a single non-absorbable polymeric Hem-o-lok ${ }^{\circledR}$ (Telefl ex Medical; New York City, USA) clip or ENDOLOOP ${ }^{\circledR}$ Ligature (ETHICON; New Jersey, USA). The appendix was divided by Ligasure just above the ligature. The appendix was removed from the left lower quadrant port and taken either altogether within the port or in a surgical glove, depending on the diameter of the appendix. The appendix specimen sizes were noted after their removal. The entire abdomen was inspected for any intra-abdominal fl uid and irrigated vigorously. Subsequent to bleeding control, a Jackson-Pratt drain was placed in right lower quadrant and the port sites were closed. At the end of the last port site closure, operating time was stopped.

All patients received a single dose of broad-spectrum intravenous antibiotic at induction of anesthesia. Postoperative antibiotic administration was dependent on operative findings and postoperative complications.

\section{Results}

Group 1 (16 male patients) and Group 2 (18 male patients) consisted of thirty patients each. The median age was 27.5 \pm 13.4 and 32.8 \pm 13.8 in Group 1 and Group 2, respectively.

The operating time was $50 \pm 10.6$ minutes for Group 1 and 
$40 \pm 12.6$ minutes for Group 2. Moreover, appendiceal base closure time was found $187 \pm 102$ seconds and $69 \pm 30$ seconds in Group 1 and 2, respectively.

Appendix diameters were measured as $10 \pm 4.1 \mathrm{~mm}$ (Group 1 ) and $7.5 \pm 2.4 \mathrm{~mm}$ (Group 2). Hospital stay was found $53 \pm 33.4$ hours and $47.5 \pm 34$ hours in Group 1 and 2, respectively.

No perioperative or major postoperative complications were encountered.

The comparison of operating time revealed statistically significant difference $(\mathrm{t}=3.22, \mathrm{p}=0.002)$. As for the base closure time, Mann-Whitney $U$ test was used to assess the findings and the values of Group 1 were significantly longer $(z=6.388, p<0.001)$.

Remaining statistical comparisons did not reveal any significance.

\section{Discussion}

In this present study, ENDOLOOP ${ }^{\circledR}$ Ligature (ETHICON; New Jersey, USA) and Hem-o-lok ${ }^{\circledR}$ (Telefl ex Medical; New York City, USA) polymeric clips were compared regarding their use in laparoscopic appendectomy. There was a significant difference regarding surgical time and base closure time in the favor of polymeric Hem-o-lok Group.

During LA, base closure of the appendix is performed by different manners including intra-abdominal knotting, extracorporeal knotting, ENDOLOOP ${ }^{\circledR}$ Ligature(ETHICON; New Jersey, USA), endoclip, polymeric clip, staplers, and etc. ${ }^{[6]}$ All techniques have some advantages and disadvantages related to the material itself or technique. ${ }^{[7]}$ Safety, application comfort, foreign body effects of the materials, operating time, and etc. are t key points defining the variety of the methods. The ideal method would be the fastest and safest way to close the base of the appendix associated with no long term complications.

Safety of the closure methods were evaluated and confirmed by numerous studies and they have shown acceptable rates of complications. ${ }^{[8-14]}$ Operating time is one of the mostly studied criteria and the application method usually defines the length of the application. Clip applications seem to be the easiest and fastest method for the closure of the base of the appendix. ${ }^{[13]}$

Endo-loops have been used in laparoscopic appendectomy for a long time in order to close the base of the appendix and divide the mesoappendix. ENDOLOOP ${ }^{\circledR}$ Ligature (ETHICON; New Jersey, USA) is designed to ensure a secure knot with relatively faster and easier way in comparison to intra-corporeal knotting technique. It has been shown that it is a practical and safe way to reach this goal. ${ }^{[12]}$ Polymeric clips are used to close the vessels in laparoscopic surgery and the base of the appendix in LA. ${ }^{[7,8,15,16]}$ The clip has a locking tip providing a very secure clipping.

The applications of polymeric Hem-o-lok ${ }^{\circledR}$ (Telefl ex Medical; New York City, USA) clips and endo-loops were previously studied in a rat model by Delibegovic et al. regarding their tissue reactions in the intestine. ${ }^{[15]}$ The results of the study revealed both materials had acceptable tissue foreign body reaction and tissue reaction degree of the polymeric clips were slightly less than endo-loops. Moreover, the use of polymeric clips has been found to be safe and effective by the study of Hanssen et al. with a special emphasize on cost effectiveness. ${ }^{[16]}$

Another related study is from Hue et al., comparing ENDOLOOP $^{\circledR}$ Ligature (ETHICON; New Jersey, USA) and polymeric clip in LA. ${ }^{[17]}$ The authors have found no statistically significant difference between these two techniques regarding the feasibility and safety of the operation. The operating time-which was found to be significantly shorter in our study-hasn't been assessed in this report unfortunately.

Our results showed superior results of the polymeric clip applications regarding technical comfort and operating time. More clinical studies are needed regarding especially the long term safety of this method in order to reach an ultimate conclusion.

\section{References}

1. Chung RS, Rowland DY, Li P, Diaz J. A meta-analysis of randomized controlled trials of laparoscopic versus conventional appendectomy. Am J Surg 1999;177:250-6.

2. Golub R, Siddiqui F, Pohl D. Laparoscopic versus open appendectomy: a metaanalysis. J Am Coll Surg 1998;186:545-53.

3. Wei B, Qi CL, Chen TF, Zheng ZH, Huang JL, Hu BG, et al. Laparoscopic versus open appendectomy for acute appendicitis: a metaanalysis. Surg Endosc 2011;25:1199-208.

4. Markides G, Subar D, Riyad K. Laparoscopic versus open appendectomy in adults with complicated appendicitis: systematic review and meta-analysis. World J Surg 2010;34:2026-40.

5. Markar SR, Penna M, Harris A. Laparoscopic approach to appendectomy reduces the incidence of short- and long-term post-operative bowel obstruction: systematic review and pooled analysis. J Gastrointest Surg 2014;18:1683-92.

6. Gomes CA, Nunes TA, Soares C Jr, Gomes CC. The appendi- 
ceal stump closure during laparoscopy: historical, surgical, and future perspectives. Surg Laparosc Endosc Percutan Tech 2012;22:1-4.

7. Gorter RR, Heij HA, Eker HH, Kazemier G. Laparoscopic appendectomy: State of the art. Tailored approach to the application of laparoscopic appendectomy? Best Pract Res Clin Gastroenterol 2014;28:211-24.

8. Sahm M, Kube R, Schmidt S, Ritter C, Pross M, Lippert H. Current analysis of endoloops in appendiceal stump closure. Surg Endosc 2011;25:124-9.

9. Alis H, Gonenc M, Deniztas C, Kapan S, Turhan AN. Metal endoclips for the closure of the appendiceal stump in laparoscopic appendectomy. Tech Coloproctol 2012;16:139-41.

10. Arcovedo R, Barrera $H$, Reyes HS. Securing the appendiceal stump with the Gea extracorporeal sliding knot during laparoscopic appendectomy is safe and economical. Surg Endosc 2007;21:1764-7.

11. Kazemier G, in't Hof KH, Saad S, Bonjer HJ, Sauerland S. Securing the appendiceal stump in laparoscopic appendectomy: evidence for routine stapling? Surg Endosc 2006;20:1473-6.

12. Beldi G, Muggli K, Helbling C, Schlumpf R. Laparoscopic ap- pendectomy using endoloops: a prospective, randomized clinical trial. Surg Endosc 2004;18:749-50.

13. Ates $M$, Dirican A, Ince V, Ara C, Isik B, Yilmaz S. Comparison of intracorporeal knot-tying suture (polyglactin) and titanium endoclips in laparoscopic appendiceal stump closure: a prospective randomized study. Surg Laparosc Endosc Percutan Tech 2012;22:226-31.

14. Rickert A, Bönninghoff R, Post S, Walz M, Runkel N, Kienle P. Appendix stump closure with titanium clips in laparoscopic appendectomy. Langenbecks Arch Surg 2012;397:327-31.

15. Delibegović S, Iljazović E, Katica M, Koluh A. Tissue reaction to absorbable endoloop, nonabsorbable titanium staples, and polymer Hem-o-lok clip after laparoscopic appendectomy. JSLS 2011;15:70-6.

16. Hanssen A, Plotnikov S, Dubois R. Laparoscopic appendectomy using a polymeric clip to close the appendicular stump. JSLS 2007;11:59-62.

17. Hue CS, Kim JS, Kim KH, Nam SH, Kim KW. The usefulness and safety of Hem-o-lok clips for the closure of appendicular stump during laparoscopic appendectomy. J Korean Surg Soc 2013;84:27-32. 